\title{
Ectopic Pregnancy Occurred after Oocyte Intrauterine Transfer (OUT) - A Case Report -
}

\author{
AKIO KATAOKA, SHOUKO HIRAKAWA, MANAMI IWAMOTO, YUMI SAKUMURA \\ AND RYOUTA YOSHINAGA
}

Clinic of obstetrics and gynecology, Kataoka Ladies Clinic, Yatsushiro 866-0861, Japan

Received 5 July 2011, accepted 18 October 2011

Edited by DAIZOU HORI

\begin{abstract}
Summary: Although still an experimental procedure, it is hoped that oocyte intrauterine transfer (OUT) could become a convenient technique for initiating pregnancy. A 33-year old woman received OUT treatment after a period of infertility lasting for 3 years. Two weeks later the result of pregnancy test was positive, but shortly thereafter she complained of vaginal bleeding. Ultrasonography revealed a cystic lesion in her right adnexal area without any ascites. At laparotomy, a right side tubal pregnancy was confirmed. This is the first case report of ectopic pregnancy occurring after OUT. It was speculated that the OUT may have caused the tubal pregnancy. However, since the precise mechanism for embryonic implantation to the tubal epithelium is unknown, the causal relationship between OUT and tubal pregnancy remains unclear.
\end{abstract}

Key words oocyte transfer, ectopic pregnancy, invitrofertilization and embryotransfer, transvaginal hydrolaparoscopy

\section{INTRODUCTION}

Recent advances in artificial reproductive techniques (ART) have been encouraging. However, patients are usually required to make repeated visits to the hospital for follicular monitoring, hormone therapy and finally oocyte retrieval. The clinical application of ART is, therefore, expensive, time consuming, and highly stressful for the patient.

Against this backdrop, it has been suggested that oocyte intrauterine transfer (OUT) could become a convenient new procedure in the ART repertoire [1]. The results of previous studies describing same-day transfer of oocytes and spermatozoa to the uterus are promising $[2,3]$. Since actual experience with OUT is limited, however, its clinical value remains to be determined.

The fallopian tube plays an essential role in gamete transport, fertilization and the early development of the embryo [4], and damage to the fallopian tube and pelvic inflammatory diseases are usually regarded as major risks for ectopic pregnancy [5-7]. Hence, most infertile women requiring ART should have an increased risk for ectopic pregnancy. We present here the first ever report of a case of tubal pregnancy occurring after OUT, and briefly discuss the causal relationship between ART and tubal pregnancy.

\section{CASE REPORT}

A 33-year old null-gravid woman suffered from infertility lasting for 3 years. Her husband already had 3 children with a former wife, suggesting his normal reproductive capacity. Her hormonal profiles of the pituitary-gonadal axis were not remarkable. Hysterosalpigography revealed that both tubes were blocked, indicating tubal atresia. Transvaginal hydrolaparoscopy also showed perifallopian tube adhesion, presumably due to chlamydia trachomatis infection (Fig. 1 and Fig. 2). OUT was performed after obtain-

Address for correspondence: Akio Kataoka, 3-3-35 Honmachi, Yatsushiro-shi, Kumamoto, 866-0861, Japan. Tel: 81-965-32-2344 Fax: 81-965-31-1367 
ing informed consent from the patient and approval for the procedure by the institutional review board. Short-term administration of gonadotropin releasing hormone $(\mathrm{GnRH})$ agonist was used to achieve controlled ovarian stimulation. When the diameter of dormant follicles reached about $2 \mathrm{~cm}, 5,000$ IU human chorionic gonadotropin (HCG) was administered. Transvaginal oocyte retrieval was performed $35 \mathrm{~h}$ after the HCG administration. Eight oocytes were retrieved and incubated for $5 \mathrm{~h}$ in a fertilization medium

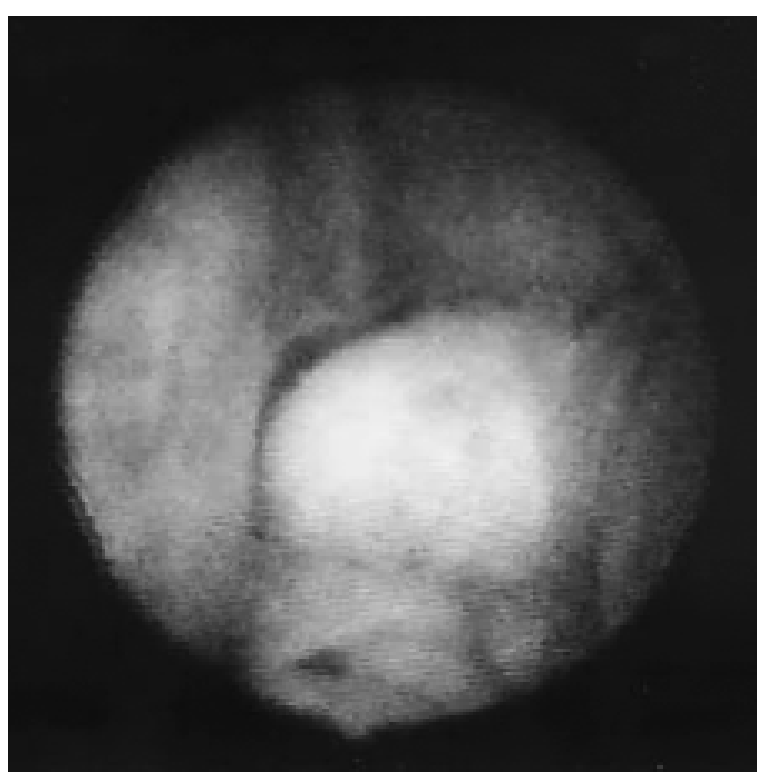

Fig. 1. Transvaginal hydrolaparoscopy, showing peritubal adhesion with sigmoid colon of the left Fallopian tube.

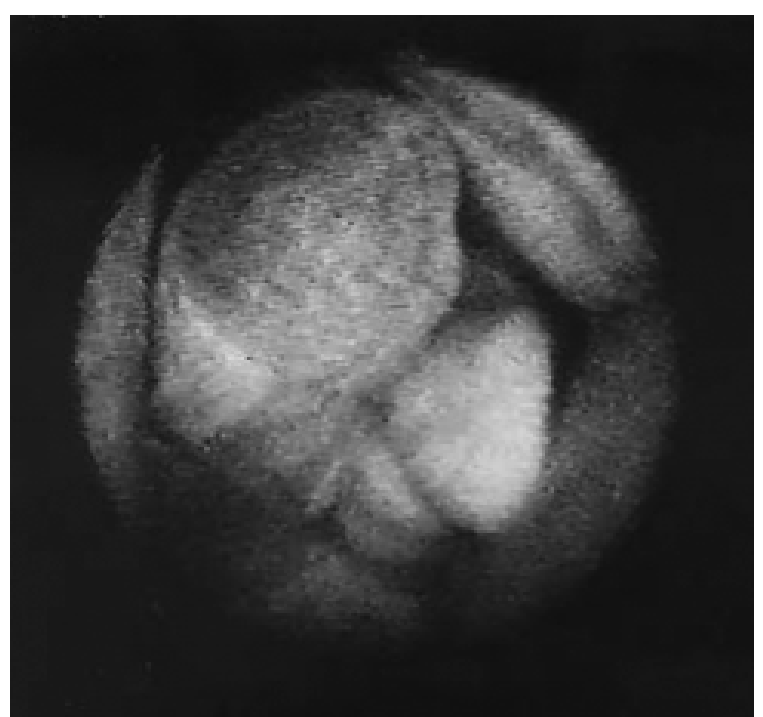

Fig. 2. Transvaginal hydrolaparoscopy. The balloon-like swelling was observed in the right side Fallopian tube.
(Quinn TM, Australia) in 5\% $\mathrm{CO}_{2}$ and $80 \%$ Nitrogen gases at $37^{\circ} \mathrm{C}$. According to the recommendations of the Japan Society of Fertility and Sterility [8], three oocytes were transferred to the uterus using an echo marked tip (ET) catheter after sexual intercourse. Fourteen days after OUT, she complained of a spotting bloody discharge, and showed serum HCG of 333 mIU. At day 21 following OUT, although the level of serum HCG elevated to $1,800 \mathrm{mIU}$, no intrauterine gestational sac could be identified. Instead, a cystic lesion was observed in her right adnexal area without ascites, and the genital bleeding continued. She finally underwent a laparotomy to confirm the right tubal pregnancy (Fig. 3 and 4), and chorionic villi were observed in the resected tissue (Fig. 5). The serum level of HCG then decreased to $2.0 \mathrm{mIU}$ within two weeks.

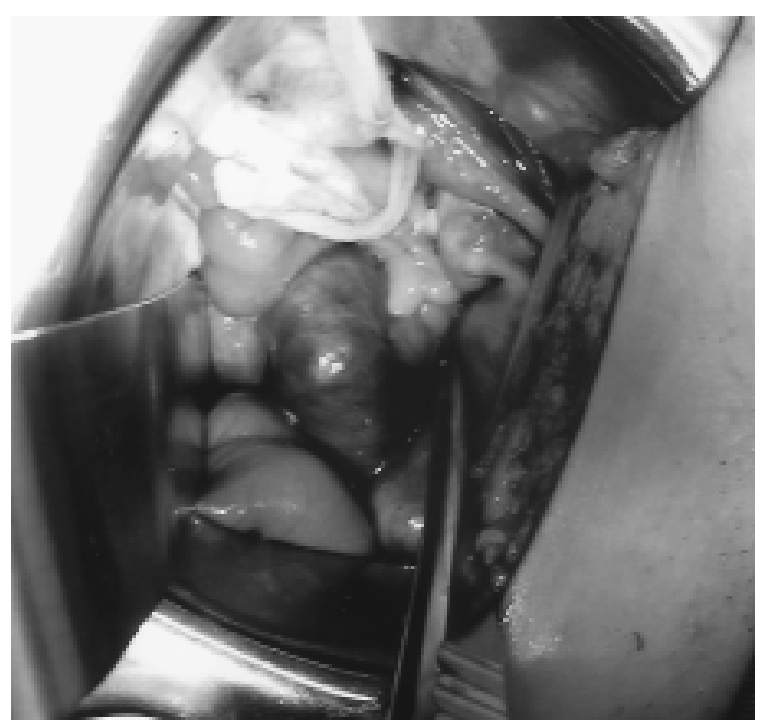

Fig. 3. At laparotomy, the Fallopian tube swelling was revealed.

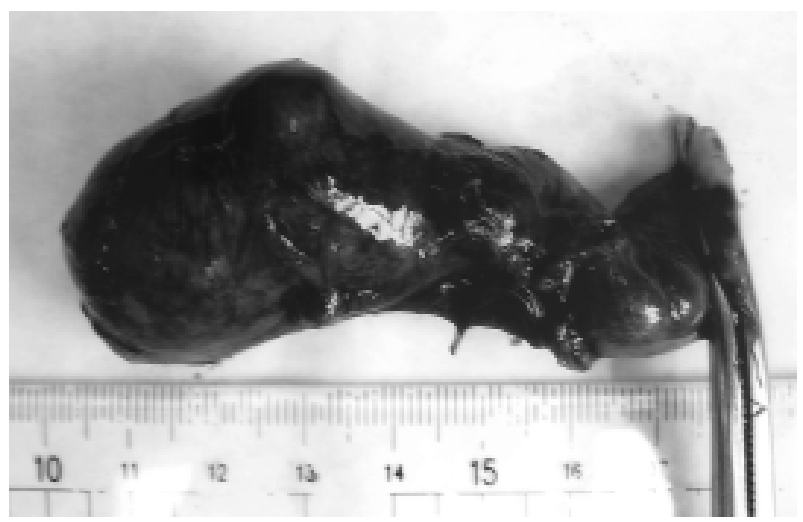

Fig. 4. In the resected tissue, the Fallopian tube was filled with coagulated blood. 


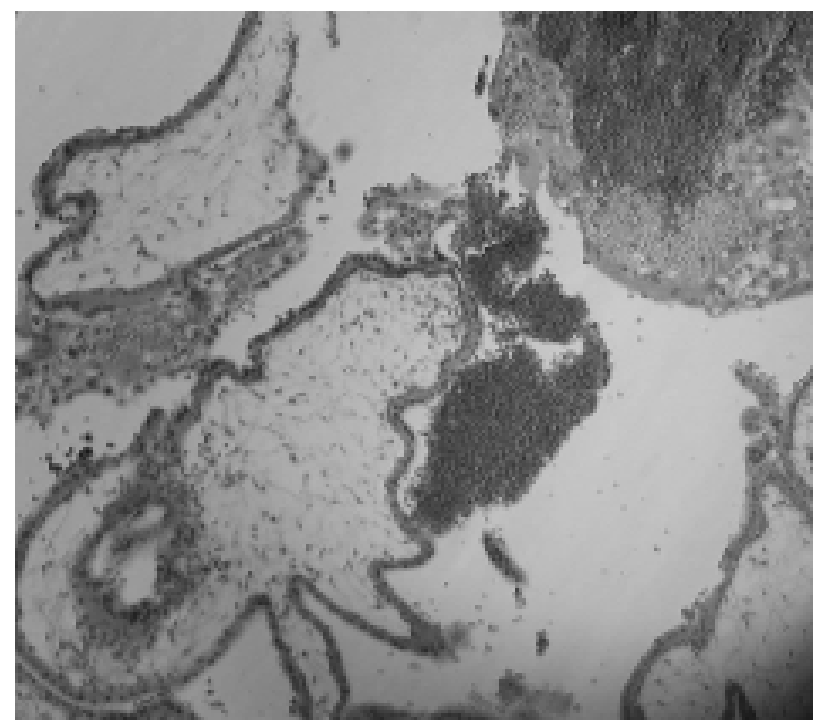

Fig. 5. In histological examination, chorionic villi were observed in the resected tissue. Original magnification $100 \times(\mathrm{HE})$

\section{DISCUSSION}

Unfortunately, our clinical trial of OUT for an infertile patient resulted in the occurrence of tubal pregnancy, and the OUT itself seemed to be a likely cause of the ectopic pregnancy. However, it remains uncertain whether the tubal pregnancy resulted from the OUT or from other factors.

A number of risk factors for ectopic pregnancy are known in humans, and ART also may increase the risk of tubal pregnancy. However, in approximately onethird [9] to one half [10] of ectopic pregnancies, no risk factors can be identified.

The era of ART began in 1978 with the first report of in vitro fertilization [11]. The earliest record of ectopic pregnancy was described by Abu al-Qasim alZahrawi, the father of modern surgery, in 963 [12], indicating that ART was not essential for the development of tubal pregnancy.

Current knowledge of the aetiology of tubal ectopic pregnancy has focused on embryo-tubal transport. Effective tubal transport of ova, sperm and embryo is a prerequisite for successful spontaneous pregnancy, and transport of the embryo through the Fallopian tube is controlled by smooth muscle contraction and cilliary beating $[13,14]$.

The interaction between the genital tract and developing embryo is not fully understood, and tubal pregnancy is currently thought to be caused by a combination of retention of the embryo within the Fallopian tube due to impaired embryo-tubal transport and alterations in the tubal environment allowing early im- plantation to occur.

Embryonic implantation consists of three related and consecutive phases: apposition, adhesion between trophoectoderm and endometrial epithelium, and invasion. In order to establish pregnancy, the initial apposition and adhesion of the blastocyst to maternal endometrium must occur in a coordinated manner.

Among animal species, tubal ectopic pregnancy would appear to be restricted to primates. Given the striking absence of tubal ectopic pregnancy in other mammals, attention has been focused on the fact that the compositions of tubal fluid and uterine fluid are similar in primates [15]. However, the roles played by tubal fluid and uterine fluid in human embryonic implantation are not clear [16].

The mechanisms responsible for ectopic pregnancy, thus, are still largely unknown. Patients considering ART should be informed of the increased risk for ectopic tubal pregnancy.

\section{REFERENCES}

1. Kato O. Oocyte intrauterine transfer. Jpn Infertlity and Sterility 2000; 45 suppl:10.

2. Craft I, Mcleod F, Green S, Djahnbakhch O, Bernard A et al. Human pregnancy following oocyte and sperm transfer to the uterus Lancet 1982; 319:1031-1033.

3. Veersema S, Bernardus RE, Veersema D, Vermeiden JP, and Schoemaker J. Pregnancy following transcervical transfer of oocyte and sperm. N. Engl. J. Med 1989; 320:1499-1501.

4. Lyons RA, Saridogan E, and Djahanbakhch O. The reproductive significance of human Fallopian tube cilia. Hum Reprod Update 2006; 363-372.

5. Saitoh M, Ishihara O, Igarashi K, Hayashi N, Taya J et al. The analysis of infertility therapy-induced ectopic pregnancy. Jpn Infertility and Sterility 2000; 45:49-53.

6. Marccus SM, and Brinsden PR. Analysis of the incidence and risk factors associated with ectopic pregnancy following in vitro fertilization and embryo transfer. Human Reprod 1995; 10:199-203.

7. Shibahara H, Suzuki T, Wada T, Ohno T, Yamauchi A et al. Bilateral tubal pregnancies after the termination of intrauterine pregnancy following gamete intrafallopian transfer. Reproductive Med and Biol 2002; 1:65-67.

8. Japan Society of Fertility and Sterility: The guide line of reproductive technology. Kanehara, Tokyo, $2^{\text {nd }}$ Ed. pp 39-53, 2003.

9. Farquhar CM. Ectopic pregnancy. Lancet 2005; 366:583591.

10. Majhi AK, Roy N, Karmakar KS, and Banerjee PK. Ectopic pregnancy - an analysis of 180 cases. J Indian Med Assoc 2007; 105:308-312.

11. Steptoe PC, and Edwards RC. Birth after reimplantation of human embryo. Lancet 1978; 2:366.

12. Buckley P, and Caine A. A high incidence of abdominal pregnancy in the Djungarian hamster (Phodopus sungorus). 
J Reprod Fertility 1979; 56:679-682.

13. Halbert SA, Tam PY, and Blandau RJ. Egg transport in the rabbit oviduct: the roles of cilia and muscle. Science 1976; 191:1052-1053.

14. Lyons RA, Saridogan E, and Djahanbakhch O. The reproductive significance of human Fallopian tube cilia. Hum Reprod Update 2006; 12:363-372.
15. Corpa JM. Ectopic pregnancy in animals and humans. Reprod 2006; 131:631-640.

16. Liu J, Li D, Cao B, Li Y-x, Herva R et al. Expression and Localization of SWAP-70 in Human Fetomaternal Interface and Placenta During Tubal Pregnancy and Normal Placentation. J Histochem Cytochem 2007; 55:701-708. 